\title{
Rapid Testing Method of New Generation Smart Substation Network Configuration
}

\author{
Lei Su ${ }^{1, a}$, Jin Wang ${ }^{1, b}$, Peng $\mathrm{Li}^{1, \mathrm{c}}$ \\ ${ }^{1}$ State Grid Hubei Electric Power Research Institute, Wuhan, 430077, China

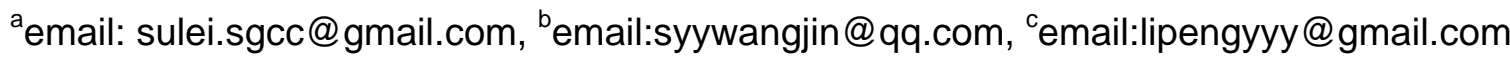

Keywords: New Generation Smart Substation; Network Configuration; Testing

\begin{abstract}
A number of popular communication traffic control protocols including virtual local area network and GMRP are applied in the new generation Smart Substation internal communication network. This paper presents a black-box testing method which does not rely on specific network configuration profile that can hopefully simplify testing requirements, and to meet the demands of smart substation operation and maintenance.
\end{abstract}

\section{Introduction}

The digitization and networking are two main characters of IEC 61850-complaint secondary devices [1]. Communication network acts as the backbone of the whole substation automation system. According to the blueprint of key technological framework of the new generation substation published by State Grid Corporation of China, MMS (Manufacturer Message Specification) as well as GOOSE (Generic Object Oriented Substation Event), SV (Sampled Values), and IEEE1588 shall share identical network by utilizing quasi-VLAN technique, to minimize network framework level and the quantity of switchers, and to maximize network efficiency. This architect requires a higher and more sophisticated network configuration so as to the method of testing

Vast amount of data flow in the substation internal network. Technologies such as VLAN and GMRP are used in this network [2-3]. Both domestic and international scholars have done numbers of research on the present logic partitioning of reliability on substation network. Paper [4] proposes an information transmission scheme based on priority tagging. Paper [5] verifies that 100M-based bandwidth can satisfy the requirement of substation automation system. However, most present research still have drawbacks: most of them only validate their study theoretically and overlook the basic need of network configuration in the field. To solve the aforementioned problem, this paper presents a rapid testing method for smart substation network configuration and uses VLAN partitioning test as an example to demonstrate this configuration profile independent "black box" testing scheme, which can be virtually utilized in any kind of logical network partitioning protocol.

\section{Module Configuration}

Figure 1 indicates the schematic diagram of rapid testing method of network configuration in the new generation smart substation. The switches are configured in accordance with the actual configuration of smart substation Ethernet switches, including $\mathrm{m}$ VLANs ( $\mathrm{m}$ is an integer and equal to or greater than 1) and each VLAN contains a set of ports. The testing device this method uses also contains full-duplex gigabit Ethernet interface and the quantity of its ports can be increase or decrease based on the need of testing requirement. In order to perform logical calculation and store its result, device comprises several modules including parameter configuration module, test packets building module, network configuration test module and report generation module. The role of each module is described as follows. 


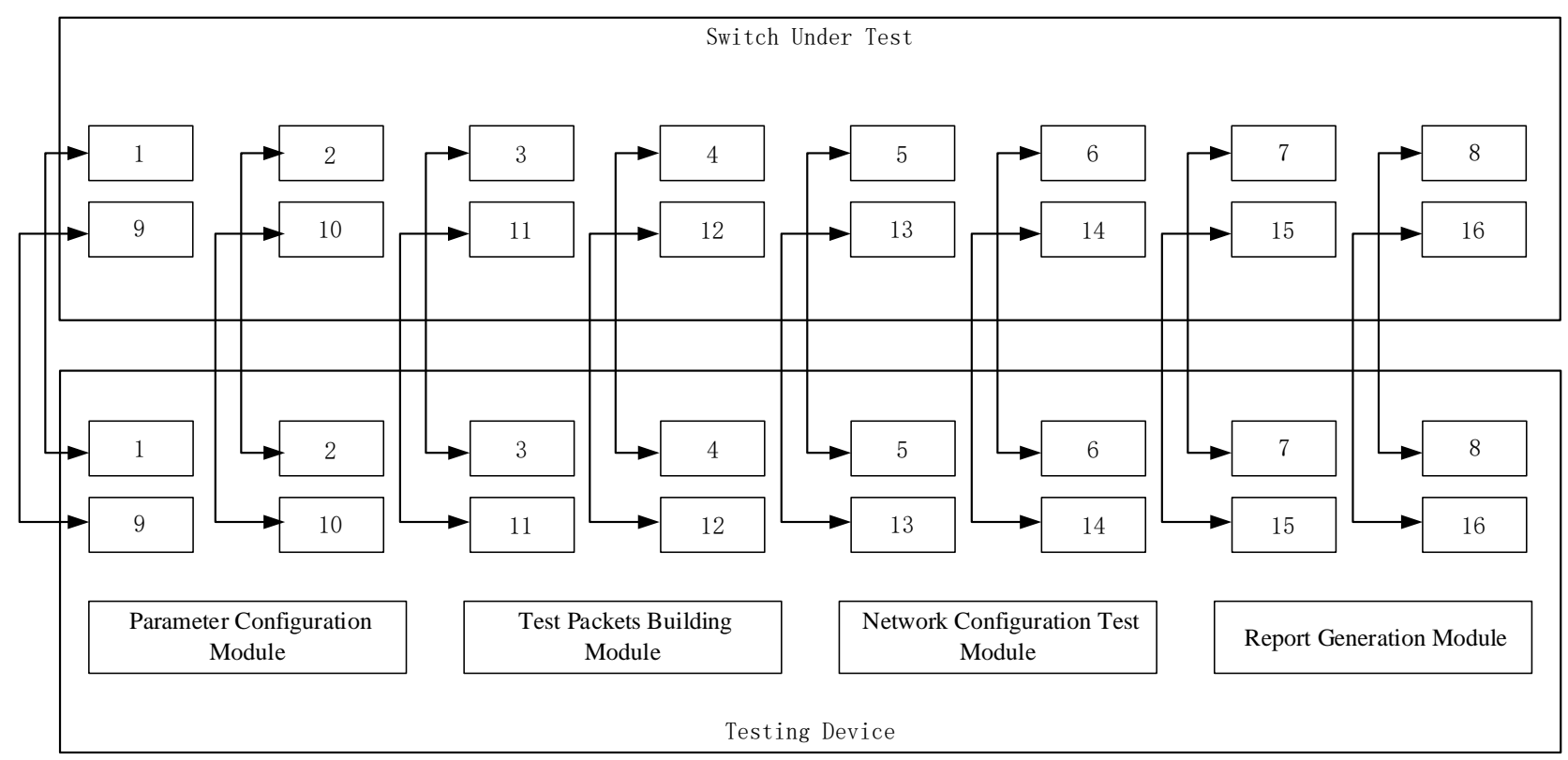

Fig.1.Schematic Diagram of Rapid Testing Method

Parameter configuration module is used to configure device testing profile which include the length of frame, test interval and the number of test that shall perform.

Test packets building module is designed to build test packets according to the smart substation network configuration regulation.

Network configuration test module is constructed for sending the test packets to certain ports of switch-under-test in accordance with test profile, and recording packets reception status of each port of the testing device,

\section{Network Configuration Testing}

\section{Generating Computing Matrix}

During the test, connect each port of testing device to the corresponding port of the switch under test (the number of ports is marked as $n$ ), and the network configuration test module will perform the following procedure: After sending testing packets from port $S_{i}(i=1 \sim n)$ of testing device to the corresponding port of the switch under test, if the packets can be received by $R_{j}(j=1 \sim n)$ ports of testing device successfully, then we can judge that $S_{i}$ and $R_{j}$ are in the same VLAN. Testing results can form an $n$ by $n$ matrix, marked as matrix $A$. The row elements of matrix $A$ are $S_{1}, S_{2} \ldots S_{n}$ and the column elements of matrix $A$ are $R_{1}, R_{2}, \ldots R_{n}$. If $S_{i}$ and $R_{j}$ are in the same VLAN, then we can mark entry $A\left[S_{i}, R_{j}\right]=1$; if not, $A\left[S_{i}, R_{j}\right]=0$. Matrix $A$ is as follow.

\section{Detection of Trunk Port}

$$
A=\left(\begin{array}{ccccc}
1 & 0 & \cdots & 0 & 1 \\
0 & 1 & \cdots & 1 & 1 \\
\vdots & \vdots & \ddots & \vdots & \vdots \\
0 & 0 & \cdots & 1 & 0 \\
0 & 0 & \cdots & 0 & 1
\end{array}\right)
$$

Calculate if Matrix $A=A^{T}$, if Matrix $A$ is equal to its transpose, or it's a symmetric matrix, then we can safely judge that there is no trunk port configured in the switch under test. If not, multiply A to a $\mathrm{n}$ by 1 column vector $\mathrm{Y}=(1,1, \ldots, 1)^{\mathrm{T}}$, the matrix product $\mathrm{AY}$ is a new $\mathrm{n}$ by $\mathrm{n}$ matrix marked as $\mathrm{Z}$, and the column or row number of elements of $\mathrm{Z}$ are identical with port number of switch under test, if the value of elements equal to 1 , then the corresponding ports are trunk ports or ports with only 
one VLAN partitioning. These ports are marked as trunk $\mathrm{k}_{1}$ to trunk $\mathrm{p}_{\mathrm{p}}$.

In matrix $A$, from row $S_{1}$ to row $S_{n}$, extracts elements that have the value of 1 , record the column numbers of those elements and use them to form new row vectors Array ${ }_{1} \sim$ Array $_{n}$., and merge those row vectors and only reserve the ones that number of entry is greater than 1 . The remaining $\mathrm{k}$ row vectors are marked as VLAN 1 to $\mathrm{VLAN}_{\mathrm{k}}$, the elements in these row vectors actually present the port number in the same VLAN partitioning.

Compare the elements in $\mathrm{VLAN}_{1} \sim \mathrm{VLAN}_{\mathrm{k}}$ with elements in trunk $\mathrm{k}_{1} \sim \operatorname{trunk}_{\mathrm{p}}$, if they share the same elements, then those trunk ports can communicate with the corresponding VLANs. If not, then those trunk ports do not communicate with those VLANs.

\section{Test Case}

Assume the VLAN configuration of an 8 ports switch is as follows:

Port 1 is set as VLAN A, Port 2, 3, 4 in VLAN B, Port 5, 6, in VLAN C, and Port 7 is trunk port for both VLAN B and VLAN C. Port 8 is trunk port for VLAN A, B, and C.

Connect each port of testing device to corresponding switch under test. When sending testing packets from port $S_{i}(i=1 \sim 8)$ of testing device to the corresponding port of switch under test, and the packets can reach $R_{j}(j=1 \sim 8)$ ports of testing device, then $S_{i}$ and $R_{j}$ are in the same VLAN. Testing results can be filled into an 8 by 8 matrix, named matrix $A$. The row entries of matrix $A$ are $S_{1}$ to $S_{8}$, and the column entries of matrix $A$ are $R_{1}$ to $R_{8}$. If $S_{i}$ and $R_{j}$ are in the same VLAN, then $A\left[S_{i}, R_{j}\right]=1$; if not, $A\left[S_{i}, R_{j}\right]=0$. After running the test, matrix $A$ is like:

$$
A=\left(\begin{array}{llllllll}
1 & 0 & 0 & 0 & 0 & 0 & 0 & 1 \\
0 & 1 & 1 & 1 & 0 & 0 & 1 & 1 \\
0 & 1 & 1 & 1 & 0 & 0 & 1 & 1 \\
0 & 1 & 1 & 1 & 0 & 0 & 1 & 1 \\
0 & 0 & 0 & 0 & 1 & 1 & 1 & 1 \\
0 & 0 & 0 & 0 & 1 & 1 & 1 & 1 \\
0 & 0 & 0 & 0 & 0 & 0 & 1 & 0 \\
0 & 0 & 0 & 0 & 0 & 0 & 0 & 1
\end{array}\right)
$$

In this test scenario, $A \neq A^{T}$ so the switch has trunk ports. Multiply $A$ to an 8 by 1 column vector $\mathrm{Y}=(1,1, \ldots, 1)^{\mathrm{T}}$, the matrix product $\mathrm{AY}$ is a new 8 by 8 matrix marked as $\mathrm{Z}$, and the column or row number of elements of $\mathrm{Z}$ are identical with port number of switch under test, if the value of elements equal to 1 , then the corresponding ports are trunk ports or ports with only one VLAN partitioning. These ports are marked as trunk ${ }_{1}$ and trunk $\mathrm{k}_{2}$.

$$
\begin{aligned}
& A \times Y=\left(\begin{array}{llllllll}
1 & 0 & 0 & 0 & 0 & 0 & 0 & 1 \\
0 & 1 & 1 & 1 & 0 & 0 & 1 & 1 \\
0 & 1 & 1 & 1 & 0 & 0 & 1 & 1 \\
0 & 1 & 1 & 1 & 0 & 0 & 1 & 1 \\
0 & 0 & 0 & 0 & 1 & 1 & 1 & 1 \\
0 & 0 & 0 & 0 & 1 & 1 & 1 & 1 \\
0 & 0 & 0 & 0 & 0 & 0 & 1 & 0 \\
0 & 0 & 0 & 0 & 0 & 0 & 0 & 1
\end{array}\right) \times\left(\begin{array}{l}
1 \\
1 \\
1 \\
1 \\
1 \\
1 \\
1 \\
1
\end{array}\right)=\left(\begin{array}{l}
2 \\
5 \\
5 \\
5 \\
4 \\
4 \\
1 \\
1
\end{array}\right)=Z \\
& \Rightarrow \operatorname{trunk} k_{1}=7 \\
& \operatorname{trunk}_{2}=8
\end{aligned}
$$

In matrix $A$, from row $S_{1}$ to row $S_{8}$, extracts elements that have the value of 1 , record the column numbers of those elements and use them to form new row vectors Array ${ }_{1} \sim$ Array $_{8}$. 


$$
A=\left(\begin{array}{llllllll}
1 & 0 & 0 & 0 & 0 & 0 & 0 & 1 \\
0 & 1 & 1 & 1 & 0 & 0 & 1 & 1 \\
0 & 1 & 1 & 1 & 0 & 0 & 1 & 1 \\
0 & 1 & 1 & 1 & 0 & 0 & 1 & 1 \\
0 & 0 & 0 & 0 & 1 & 1 & 1 & 1 \\
0 & 0 & 0 & 0 & 1 & 1 & 1 & 1 \\
0 & 0 & 0 & 0 & 0 & 0 & 1 & 0 \\
0 & 0 & 0 & 0 & 0 & 0 & 0 & 1
\end{array}\right) \quad \begin{gathered}
\text { Array }_{1}=[1,8] \\
\text { Array }_{2}=[2,3,4,7,8] \\
\text { Array }_{3}=[2,3,4,7,8] \\
\text { Array }_{4}=[2,3,4,7,8] \\
\text { Array }_{5}=[5,6,7,8] \\
\text { Array }_{6}=[5,6,7,8] \\
\text { Array }_{7}=[7] \\
\text { Array }_{8}=[8]
\end{gathered}
$$

Merge those row vectors and only reserve the ones that number of entry is greater than 1 . The remaining 3 row vectors are marked as $\mathrm{VLAN}_{1}$ to $\mathrm{VLAN}_{3}$, the elements in these row vectors actually present the port number in the same VLAN partitioning.

$$
\begin{aligned}
& V L A N_{1}=[1,8] \\
& \operatorname{VLAN}_{2}=[2,3,4,7,8] \\
& \operatorname{VLAN}_{3}=[5,6,7,8]
\end{aligned}
$$

Compare the elements in VLAN $\sim_{1} \sim \mathrm{VLAN}_{3}$ with elements in trunk ${ }_{1}$ and trunk $\mathrm{V}_{2}$, if they share the same elements, then those trunk ports can communicate with the corresponding VLANs. If not, then those trunk ports do not communicate with those VLANs

$$
\begin{aligned}
& \operatorname{Vlan}_{1}=[1] \\
& \operatorname{Vlan}_{2}=[2,3,4] \\
& \operatorname{Vlan}_{3}=[5,6]
\end{aligned}
$$

The above 3 arrays present the actual VLAN configuration in switch under test.

\section{Conclusion}

This paper presents a rapid testing method of smart substation internal VLAN partitioning which is configuration profile independent and such "black-box" testing algorithm can be achieved through basic matrix operations. Hopefully this testing method can promote productivity and overcome drawbacks of traditional testing approaches.

\section{References}

[1] Jun Yu, Xiaofu Xiong, Yuan Zhang. Research and simulation on New Reliability Measure for Digital Substation Protection System, Power System Technology [J], 2009.33(4): 28-33.

[2] Shuwen Ding. Choosing Internal Communication Network of Digital Substation Integrated Automation System, Relay [J], 2003.31(7):37-40.

[3] Tengbo Ding, Research of the Virtual Local Area Network in Smart Substation, Power System Protection and Control [J], 2012.40(1):115-119.

[4] Jianbo Xin, Xianzhong Duan, A Transfer Scheme Based on Priority-Tag in Switched Ethernet for Substation Process-Level, Power System Technology[J], 2004.28(22):26-30.

[5] Xiaobo Dou, Minqiang Hu, Zaijun Wu, Simulation Analysis on Performance of Communication Networks in Digital Substation, Power System Technology[J], 2008.32(17):99-104.

[3] Zhizhong Yin. Application of FPGA control DC motor servo system [J]. Inner Mongolia Science and Technology and Economy, 2008177 (23) 101-103.

[4] Joerg Christian Wolf, Phil Hall, Paul Robinson, Phil Culverhouse. Bioloid based Humanoid 
Soccer Robot Design, 2007.

[5]Wu Chuan-yu, He Lei-ying, Design and Realization of Instructional RPPR-Robot, Research and Exploration in Laboratory.2007, 26(10) 